\title{
Digital Curation of Online Resources among English Learners at Chinese Universities
}

\author{
Shuang ZENG | ORCID: 0000-0002-0505-2278 \\ Faculty of Foreign Languages, University of Shanghai for Science and \\ Technologyv, Shanghai, China \\ s.zeng@usst.edu.cn \\ Jingjing ZHANG | ORCID: 0000-0002-0584-534X \\ Big Data Centre for Technology-mediated Education, Beijing Normal \\ University, Beijing, China \\ jingjing.zhang@bnu.edu.cn
}

\begin{abstract}
Moving beyond the 'digital native' rhetoric, this paper investigates what Chinese undergraduates are actually doing with online resources to learn English outside the classroom. Particular attention is paid to their dealing and managing online resources in their English learning context. A sociocultural framework is adopted to understand learners' behaviours surrounding digital technology. This theoretical position guides the exploration into the contextually mediated choices and practices of English learners in the information-rich age. Data collection for this study includes semi-structured interviews with 49 undergraduates from two large Chinese universities. The data demonstrate that when it comes to digital curations of English learning resources, learners personalize online data and build towards a digital library. Yet, many online resources were rashly disposed and consumed. More importantly, the ways that learners deal with digital information and resources are largely shaped by their context of language learning. In light of the above, the paper goes on to consider the number of contextual factors that appear to constrain learners' curation of online learning resources - not least the cultural artefact of examination and the existing beliefs about language learning.
\end{abstract}




\section{Keywords}

Digital Curation - learner agency - out-of-class English Learning

\section{Introduction}

It is widely believed that the main arena of second language (L2) learning is not confined to the language classroom (Lai, 2014). Beyond the formal provision of L2 learning, good language learners are found to engage in a wide range of learning activities (Benson \& Reinders, 2011). These learners actively seek cultural products such as TV programmes, films and magazines as they intentionally or incidentally learn English (Lai, 2014). In this information-rich age, these $\mathrm{L} 2$ resources and venues are made readily available for language learners around the world (Qian \& Bax, 2017). In fact, new patterns of learning practices are seen to have emerged with online technology as the 'catalyst' or 'cause' for change, as claimed by Raith (2009, p. 289):

Web 2.o has changed language learning because speakers of a foreign language already use Web 2.0 to communicate meaning and generate content in new genres, by using the foreign language as a lingua franca.

However, discourses such as this often lack empirical reasoning in terms of what L2 learners actually do outside the classroom and thus the actual change of L2 practices in the digital age. These widely embraced assumptions are led by the techno-deterministic envisage that 'digital native' learners are changed by the 'digital'. That is, the new generation of 'digital native' or 'modern' (language) learners behaves differently as they grow up and live a life with online technology. In this line of thinking, the ways they dealt with digital resources could be spectacular and innovative.

At the same time, the term 'digital curation' has been used in literature to capture these new forms of learning practices. Although the term per se has stimulated a great deal of debate, it is widely used as an "umbrella concept for digital preservation, data curation, electronic records, and digital assets management" (Dallas, 2017, p. 1). Digital curation is considered to be important to learning, as learners have been theoretically positioned at the centre of their learning and decision-making (Potter \& McDougall, 2017). This 'goodness of fit' between learning theories and the creation-oriented practices have led to the assumption that learners are innovative when dealing with digital resources out-of-class (Jenkins et al., 2006). However, there still lacks empirical studies 
which aim to demystify and understand digital curations outside the classroom (Poole, 2017). As such, the present study aims to uncover how do English learners deal and manage the information and resources obtained from the Internet? And why do they choose to do so? This study adopts the broad-brush description of digital curation mentioned above, as the empirical study does not limit itself to any specific form of digital curation, but is essentially open to the behavioral patterns emerged from dealing and managing the digital resources for L2 learning related purposes. It is hoped that this paper can contribute to our understanding of how Chinese undergraduates curate digital resources for English learning related activities, and thus the digital choices and behavior of young people in the age of information overload (Zhang et al., 2019).

\section{2 \\ Literature Review}

\subsection{Digital Curation}

Empirical research and discussion on digital curation is often concerned with creation-driven pedagogies and media literacy competences (e.g. Dooly \& O'Dowd, 2012; Godwin-Jones, 2015). For example, Kurek and Hauck (2014) acknowledged the 'participatory cultures' of young people and proposed to adopt a three-tiered training framework to improve learners' digital literacy:

Many young learners have embraced what has been termed online "participatory cultures” (Jenkins, Clinton, Purushotma, Robison, \& Weigel, 2006) and know how to build their online presence through social networking sites, avatars, audio/video casts, mash-ups, and/or by taking part in online gaming. Yet, harnessing the full potential of digital offerings requires strategic action guided by a personally unique blend of competences on a technical, cognitive, social, communicative, and even personality level. Therefore, it seems highly unlikely that multiliteracy skills for more formal educational purposes such as language acquisition can be obtained by learners through informal and uninformed technology practices (p. 123).

In line with this proposal, Mihailidis (2015) conducted a case study of 47 undergraduates from two American institutions. The students were required to curate essays in 60 minutes through the social curation platform Storify. Upon completion of their essays, students were directed to complete an online questionnaire which was concerned with students' curation experiences. 
The results showed that students were largely replicating their social network behavior to curation for educational purposes. They "reposting," "sharing," "searching," "interacting," and "remixing," when dealing and negotiating information and resources online (p. 452). Their learning experiences were perceived to be positive. Mihailidis (2015) concluded by arguing "curation as central to the development of digital and media literacy competencies that are student-driven, creation-driven" (p. 453). Chen (2017) explored the potentials of curated materials for improving English listening skills among 27 English majored undergraduates in Taiwan. To foster learner-directed learning through extensive listening, Chen (2017) provided curated listening materials to English learners so as to facilitate their choices of online resources and information. This research demonstrated the potential of digital curation on improving pedagogies and inducing learning innovations.

What we can learn from all these studies and discussions is that learners today are more proficient with digital technology and exercise their individual agency when engaging with digital resources. Digital curation is important to learner-centered and creation-driven pedagogy, but still, learners need to improve their digital literacies so as to harness the full potential of digital technology. Despite these insights, existing literature provides insufficient account of how learners as agents make decisions and curate the over-abundant information and resources out-of-class. Accordingly, the focus of this paper lies at the heart of exploring and understanding out-of-class digital curation among L2 learners. With learners themselves offering the best insight, this initiative can help identify the possible change of L2 practices and uncover the barriers to such a change. As such, the realistic picture projected by this research will add to our understandings of out-of-class language learning with digital technology, as well as the curative behavior of young people in the informationrich age.

\subsection{Sociocultural Theory and Learner Agency}

The present study takes the sociocultural perspective when seeking to understand digital behavior of L2 learners. One central concept concerning sociocultural theory is that human mind is mediated (Lantolf, 2000). In particular, human activity is perceived as mediated by physical and symbolic tools. Physical tools indicate social agents with their own beliefs and purposes, as well as material articles that are culturally defined. Symbolic tools are more intangible and exemplified by the property of language (Lantolf, 2000). Here, language regulates our thoughts in the form of self-directed and inner speech (see Vygotsky, 2012). From this line of thinking, humans use these socially and culturally constructed tools to mediate and regulate their relationships with the 
social world. Accordingly, when looking at learners' curations of digital resources, mediation can be perceived to take place at two levels. At the microlevel, curations are mediated by the adoption of technology. Here, innovative learning behavior might emerge owing to the assistance of the technology. At the macro-level, learners' actions and decisions are mediated by the social worlds. Here, the focus is on how properties of the social worlds mediate learner decisions and practices. This provides foundation to explain some of the learning behavior of the 'digital youth'.

Theorized as such, this inquiry views language learners as agents, who make choices as to how to curate digital resources for language learning related purposes. Our view of agency has two dimensions: the capacity to act on the world, and the will and beliefs in learning. In this vein, learner agency in this paper involves the capacity to manipulate digital resources and use them to produce robust language learning opportunities and/or environments. On the other hand, when it comes to digital curations, the sociocultural perspective also leads us to consider socially and historically constructed subjectivity in relation to English learning. These interpretations encourage us to take language learners as contextually shaped agents who have been socialized into particular learning beliefs/motives. This can be identified in language learners' accounts of the ways they curate digital resources, as well as their English learning motivations. As such, this inquiry endeavours to go beyond the discourses of 'digital natives' and examines the role of social and cultural context in terms of framing learners' behaviours surrounding digital learning resources online.

\section{Methods: participants and Context of Study}

The data reported in this paper comes from a larger research which focuses on L2 learners and their use of technology beyond the classroom at Chinese universities (for details, see Zeng, 2018). The larger research adopted a mixed sequential approach to investigate out-of-class language learning. Particularly, the larger research has two distinct phases. The first-and survey phase involved 1,485 undergraduates from two large Chinese universities. The survey study was expected to provide a broad stroke of the researched phenomenon. This helped to construct the second- and interview phase, which aimed to refine and expand the data collected from the quantitative phase, as well as to explore the research problem in more depth (Creswell, 2009). A total of 49 participants have been involved in the in-depth interview study. The interviewees were purposefully selected from the survey phase, with the aim to involve both 
the participants who were enthusiastic about technology use and those who chose to engage lightly with the digital technology when it comes to English learning. As this paper attempts to particularly explore and understand the learners' curations of digital resources for English learning related purposes, data from the in-depth interviews were used to analyze the research problem. As Lai (2014, p. 119) noted: "to understand the nuances of this phenomenon (out-of-class English learning), qualitative studies are very much needed to provide further insights into the nature of the self-directed technology use for language learning...."

As such, participants of the present study came from various learning disciplines and were in different years of undergraduate study. They were asked open-ended questions during the interviews, regarding the features of English learning after class, how do they manage online resources, how to they use digital learning materials, and the reasons underlying their digital behaviour and decisions. Accordingly, patterns of digital curation were captured through learners' accounts on how they chose, managed and consumed online resources for English learning related purposes. Thematic content analysis was used to examine and code the qualitative data collected.

It is worth noting that all of the participants have an over decade of experiences of English learning (see Figure 1). Thus, they might have been acculturated into a particular way of English learning by the time they were interviewed. It should be noted that the artefact of examination is prominent in the Chinese schooling system. To secure the Bachelor's degree, all the non-English majored students are required to continue their English study and pass the standardized English test CET4. The goal is that undergraduates will be able to meet the English level specified in the National College English Teaching Syl-

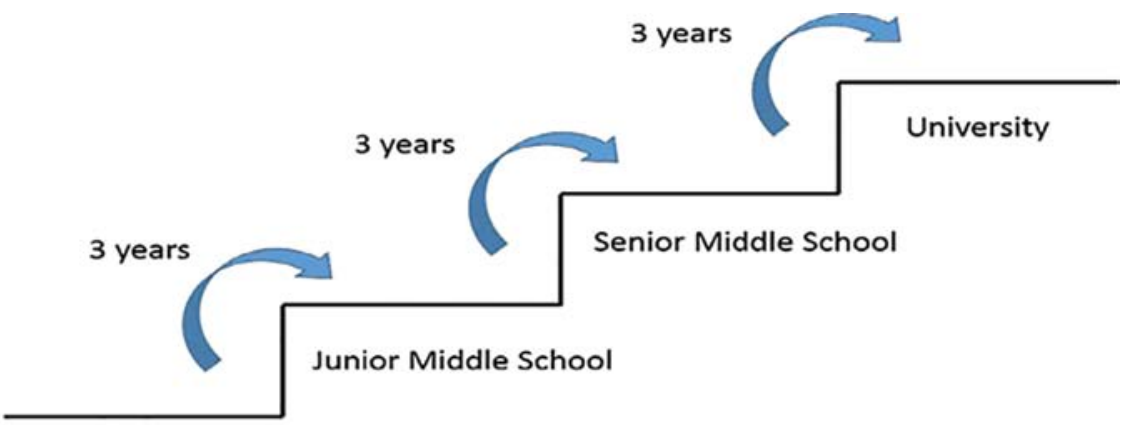

Upper Primary School

FIGURE 1 Years of English study before attending university (from Zeng, 2018, p. 9) 
labuses by the time of graduation. English majors have to pass TEM 4 but often aim for TEM 8. TEM 4 is seen as "an annual test mandatory for English-major undergraduates in China", and TEM 8 as "the uppermost test for four-year English major students" (Zeng, 2018, p. 10). There are also mid-term and final English examinations each term.

\section{Results: the Maintenance and Consumption of Digital Resources}

This section reports on how the 49 participants deal and manage digital resources for English learning. Particularly a group of learners were found to curate online resources and information in a number of ways - i.e. downloading, sharing, printing and note-taking. In other cases, however, online resources were rashly disposed and consumed. Alternatively, much of this information was visited once and not maintained for future references. Accordingly, four themes have emerged and constituted the dynamic picture of learners' engagements with online resources. In light of the interview data, these digital practices are often mediated by a range of variables including students' existing learning orientations and behaviour, as well as their situational circumstances.

\subsection{Personalising Online Data}

It was found that a minority of students had curated the content of the online materials obtained. For instance, as noted earlier, participant 29 described herself as a 'downloading expert' who had made extensive uses of online multimedia for her English learning. She also talked confidently about the computer during the interview and noted to 'mash-up' some of these materials for her learning needs:

I am a little bored lately, so I got several movie scripts. And I have to say that I'm kind of good at computers. I used 'QQ cinephile' or movie maker to get the audio tracks of the (English) movies. Then I put them in my MP 3 player and listen to them when I couldn't fall asleep at night (participant 29, engineering, female, year3).

Such curations of digital resources had certainly personalised the digital materials and enabled a more regular use of these resources, thus increasing the opportunities for English exposure. She also seemed to enjoy this sense of flexibility and control of learning materials. However, the instance of 'mashing-up' WELL resources was by no means a commonplace. More frequently practiced was the note-taking of online content for learning initiatives. Interestingly, a 
number of students claimed to document what they perceived as 'good' English encountered online on their notebooks, as demonstrated by participant 12:

Interviewee: I think at most I learn (from American TV series) about spoken English and intonations, and how they (westerners) express themselves. No matter in the past or at present, I like to write down those good expressions and sentences.

Interviewer: why on notebook? Why not your online space for instance?

Interviewee: If I put them on my (online) space, I can't read them whenever I want to. Do you think it's realistic to open my computer and go to my space to go over them when I am on the toilet? But with a small notebook I can.

Interviewer: Why don't you use your mobile phone?

Interviewee: I seldom use mobile phone for online surfing, because it has some limitations. ... Although you can browse web pages using mobile phone, the screen is very small, and you can't read some information. I always think it's not convenient, but it's portable.

(Teaching Chinese as a foreign language, female, year 1)

Clearly such digital curation and the 'pervasive' use of curated information could hardly be said as 'new' in the student's learning efforts. However, her remarks do begin to uncover the barriers faced by mobile technologies in facilitating the 'ubiquitous' language learning, as echoed by participant 4: "all the stuff in my mobile phone those for listening. I rarely put stuff to read in it, after all it's not good to the eye" (cultural heritage and museum, female, year 1). Put aside these technological 'limitations', it seemed that the technology was easily abandoned once it failed to support established learning behaviour. As such the student's online curation was not technologically driven but to a large extent shaped by her existing learning behaviour. A similar story of taking notes of digital resources was elaborated by participant 2 :

Er, when I read books sometimes I like to write down the good stuff, so as to accumulate those good English expressions. And I also share stuff about CET4 and 6 in (online) space and Renren. When I come across 
these materials, I'll write down the phrases I think useful and study them when I go out in the morning (Chemistry Education, female, year 1).

Admittedly, the efforts of note-taking did have tailored the digital information to the individual interests of the student. However, what became clear from this student was that this seemingly original curation of online content was only replicating the student's practices with paper-based materials. Moreover, it seemed that such curation was exercised in an attempt to serving her established learning behaviour. Even so, the instances of curating the digital content per se were merely a handful.

\subsection{Building Towards a Digital Library}

Whilst the curation of online content was only found within a small group of students, the applications of downloading, sharing and printing were commonly practiced for various learning initiatives. In particular, when questioned whether their language learning would be influenced if the Internet became unavailable, some students tended to give a negative answer providing they could use the online resources maintained in their laptops/PCs. That is, these students have downloaded and stored these online resources, and in this way constructed a digital repository of their own for language learning, as reflected in the following excerpt:

I don't think it (learning English without Internet) will have a big impact (over my learning)....Because how to put it, my computer has already stored some resources, if I don't have internet this month, I would simply repeatedly read the resources I've got. Perhaps this is even better than only studying these (materials) once and then leaving them there. (participant 41, engineering, male, year 2).

This kind of digital maintenance was also witnessed in other occasions. One typical example was demonstrated by participant 36 , whose curations of the online multimedia had well served his learning needs:

In fact I've downloaded a lot of learning materials. I've got last year's в вС news, I downloaded them all to my computer, and if I like I can just copy and paste these files to my MP3 player... I've been listening to (these) news since winter holiday. I make sure to listen to two or three pieces everyday (English, male, year 1). 
Furthermore, 'sharing' as a form of online storage was found especially popular among the students. Such curation, which usually took place on the social web, was seen as a means to store 'useful' information for future references. For example, when it comes to learning English outside of the classroom, participant 7 seemed to be rather passive and task-oriented, as revealed below:

Interviewer: So how do you learn English after class?

Interviewee: (laugh)

Interviewer: You think you haven't learned at all?

Interviewee: I do my homework, that's almost it, just do some exercises and study grammar books.

(English education, female, year 2)

As such completing class assignments seemed to constitute an important part of the student's English learning. In this sense she demonstrated how she curated online resources to aid her academic tasks: "But sometimes when I can't find any resources when I am writing essays, I'll go to my (online) space. Recently our teacher asked us to write an essay, many stuff I used (to complete it) came from my online space". Here the social web seemed to be maintained as a resource tank for future tasks. Participant 7 was not an isolated case. Indeed, as discussed earlier, a majority of the interviewees described engaging in exam-oriented learning activities. Correspondingly, many of these students signified a serious and pragmatic approach in curating these exam-centred resources and information - as in this example:

When I got useful materials I would download them directly to my computer. As to those mock tests I downloaded, I would delete them after I was done with the exams...they (peers) shared CET4 vocabulary lists (on Renren, a social networking site), and some stuff like 'these vocabularies can be memorised like this'. I usually marked them as my favourite. When the exams are coming, I'll print them out and study them for the finals...I'll delete them afterwards (participant 39, English, female, year 1$)$.

Clearly these curations were exam-oriented and went beyond 'building towards a digital library'. Additionally, these practices demonstrated how the student further curated the materials deposited online. Here two notable aspects have emerged: (a). The digital data was stored in two distinct places - the student had downloaded and shared online resources respectively in her 
laptop/PC and on the social web. More specifically, the students seemed to only 'share' the peer-shared information online. Thus the social web could only be said as a convenient and temporary storage for peer-shared resources. (b) More prominent here, however, was the student's attempts to transfer digital data to paper-based materials. This strong propensity for texts on paper was also manifested in other cases, perhaps because the resources curated this way could better support students' accustomed learning patterns, as recurring in the following remarks:

After all the stuff (language learning resources) I bought was paper-based because you can read them repeatedly. I'll even print out the resources I found online (participant 10, chemistry education, female, year 2).

A few months ago, I printed out all the resources I shared, and studied them at night and read them in the morning (participant 6, engineering, year 1$)$.

\subsection{Digital Curations by Situational Circumstances}

Aside from the micro-level elements of students' individual learning characteristics, the macro-level of situational circumstances also had an impact on students' curations of digital resources. For instance, as the university in Nanjing limited the Internet use among its first year students, some interviewees in such a circumstance reported a slightly different curation of online resources. One case in point was displayed by participant 18 , who was a first year engineering student from Nanjing. This student was a motivated English learner and noted how she curated online resources for language learning:

Because although I have a computer in my room, I don't have Internet access there. So I could go to the library first and downloaded the materials to my USB and take my time to read them in my dorm, or I can simply print them out.

It could be seen here that the institutional constraint of Internet access did have an effect on students' digital curation - the process was slightly more complex than that of discussed earlier. Yet, these patterns of curations were not essentially different - they mainly involve downloading and printing online resources. Perhaps this parallel was attributed to the fact that even with an 
easy Internet access, many interviewees had curated the online data in a way that it could be consumed offline. Moreover, the digital curations of some students were also influenced by their specific and immediate circumstances, as revealed in the following cases:

Because sometimes when I am downloading (English learning) materials, the internet is a bit slow. So sometimes I found it very troublesome. I don't like downloading resources, as far as English (learning) concerned.... Because the internet is very slow, I often copy and paste the materials once I found them online. I like to get (English learning) materials this way. (participant 46 , chemistry education, female, year 1 )

I've (also) used online storage several times. Some printing places won't allow you to use USB, because they fear your USB may contain virus. So I uploaded these materials (mock papers) to online storage and print them out from there. (participant 36, English, male, year 1)

As we have seen, the digital curations of some individuals were structured in such a way as to circumvent certain technological barriers. Thus their actual processes of data maintenance and storage were immensely variable but what was clear was that they had had the opportunity to develop 'autonomy' in dealing and managing the online resources obtained. Yet, these digital curations, in themselves we found, were unlikely to have essential differences. Perhaps this was because most of the online resources were curated to facilitate students' existing learning orientations and behaviour, as discussed in the previous subsection.

\subsection{Rash Disposition of Digital Resources}

Of course much learning information online was not even curated and thus used once only. However, at times even the curated learning resources were not necessarily digested or revisited. Indeed 'fast food' and 'instant noodles' were employed by a number of students to describe their consumptions of learning resources online - as in the example of participant 36 :

Learning (English) with the web is like eating instant noodles, it's quick, and you forget it easily, it's not like the book we learn, you will read it several times. The teacher will explain the contents and you would try to memorise the beautifully written paragraphs.... for example, I mentioned sharing on Renren just now, it's like you take a glimpse at a material, and 
think it's good, and then you share it, so in the end you have shared numerous learning materials but never seriously read them.

Much information on Renren is trash...because there are too many articles and they are rashly constructed and shared.... And this repeated (online) sharing doesn't have much significance. (English, male, year 1)

As can be seen here, while this student attached a great importance to formal learning materials, he took a completely different approach towards the resources shared on the social web. Such divergence, comparatively, might partially due to the less authoritative and guided nature of online learning materials. Another example in this vein was accounted by participant 41: "I personally think the resources online have updated too fast, and then I can't learn them precisely" (engineering, male, year 2). Here the two students had both attributed their insufficient attention to the overwhelming and assorted nature of online data. From another viewpoint, however, they seemed to be still indulged in a teacher-centred learning after class and lack the capacity or impetus to cope with the complexity of online resources.

Additionally, participant 36 's accusation of online sharing might also contribute to the depicted phenomenon of 'eating instant noodles'. In his opinion, online sharing was not derived from careful planning or contemplation and thus assumed less learning significance. Even the student himself only 'took a glimpse' before sharing the learning materials encountered.

Such attitudes towards online sharing were also reflected in some other interviews. For these students online sharing did not seem to be a serious means for maintaining learning resources, but a channel to pass information, a learning consolation, an legitimate act on sNs and finally just for 'fun'. This was revealed in the following remarks:

I share because sharing is fun, or I think it's useful to others (Participant 27, Math, male, year 2).

I think sNs has a 'fast food' phenomenon, including microblogs, I just browse the information (on them) and immediately share the stuff I found interesting, and seldom come back to these resources (Participant 44, Palace Literature, female, year 2).

It makes you feel like you've shared English learning resources so you've tried to learn English...I've shared many resources, but I don't bother to memorize them (Participant 3, Sociology, female, year 3). 
It could be seen from these instances that the intentions of online sharing were not necessarily learning related. Instead, such behaviour seemed to fit into the collective and entertaining culture of the social web. Despite so, this platform had somehow blurred the boundaries drawn between learning and 'having fun'. After all, notwithstanding the various motives of online sharing, it had helped to maintain and disseminate learning resources at its own endeavour.

\section{$5 \quad$ Discussion}

The lack of digital curations or innovative digital practices is not a conventional topic to explore in the field of language learning and technology (Kaya, 2013). As such, the findings reported in this paper are also compared with the literature in the wider field of educational technology. As noted at the outset of this paper, language learners are assumed to exercise their agency and innovatively curate online resources (e.g. Raith, 2009; Kurek \& Hauck, 2014). However, the empirical data has demonstrated a somewhat instrumental and unspectacular picture of digital curations in out-of-class English learning. Here, unspectacular primarily indicates that learners mainly focused on archiving information, and did not recreate or produce new information, or curated information for communication related purposes. Admittedly, a few tech-savvy learners mashed-up online resources and attempted to personalize online data for their learning needs. Yet, many learners were found to curate online resources in the way that replicated their practices with traditional paperbased resources. For example, learners did not show a preference over information in the digital format and noted down the important information on paper for future references. Moreover, the empirical research found that L2 learners tried to build towards their own digital archives. They downloaded resources from the Internet, storing them in their electronic toolkits, or in their social networking sites. However, they often printed out these learning resources for consumption. These descriptions derived from the empirical research have challenged our understanding of the so-called 'digital native' learners (Jenkins, 2006).

L2 learners are found to be agentive. The interesting cases of 'issues and solutions' have certainly revealed the ability of learners to 'act on the world' when facing the technical barriers in their immediate context. Yet, these cases have also confirmed the often unspectacular use of online resources among the L2 learners (e.g. passive consumption of information). It is likely that the issues of Internet speed and access would be of greater concern if L2 learners 
need to engage in more creation-centered activities or use more sophisticated tools for digital curation. In such cases, L2 learners might lack the skills and 'know-how', which can be further explored. Finally, it should be noted that much of the digital resources were rashly disposed by L2 learners. These decisions and practices were found to be largely linked to learners' existing beliefs about English learning.

To sum up, this inquiry found that the priority of the participants was to 'getting by' examinations, rather than 'getting on' with their English learning. Therefore, 'digital native' learners' management of digital resources were not as spectacular or groundbreaking as assumed in some existing literature. L2 Learners' digital behavior is evidently subject to the context and culture of language learning, even if empowered with 'transformative' technology in the information-rich age.

\subsection{Context, Agency and Digital Curation}

The existing literature explained the barriers to innovative curation of digital resources from the perspective of digital literacy (e.g. Mihailidis, 2015). However, this inquiry argues that the phenomenon of digital curation is also linked to a combination of contextual factors. Firstly, the phenomenon of digital curations is related to learners' context of technological infrastructure, as evidently demonstrated in the cases of how learners curated resources to avoid technical barriers. Secondly, one prominent reason underlying the lack of innovative curation of digital data was that learners had little interest to do so. In the light of the interview data, learner decisions as such were contextually mediated. In this study, as demonstrated earlier, many of the participants were learning English because they were required to do so. The prominent goal of their English learning was to pass or excel in the exams. In other words, they regarded English as a subject to learn, rather than a communicative system to acquire. These views and motivations about English learning were mediated by their past learning experiences and the exam-orientated learning reality. In other words, their current learning beliefs and patterns have limited their understanding of what English learning is and thus what they should do with digital resources for English learning related purposes.

These contextually shaped learning perspectives, together with the pressure from high-stakes exams, have driven most of the participants to continue their L2 journey using traditional methods of archiving learning resources, or at best putting some of this 'old wine' in the 'new bottles' of digital curation. This to some extent fits Crook's (2012, p. 64) observation of digital practices in education: 
We are living in a time of participatory tools, participatory attitudes and participatory aspirations; yet educational practice does not seem to be easily bringing these elements into an expected alignment.

As discussed at the outset of this paper, 'digital youth' could be innovative learners. However, emerging online technologies cannot be taken as the remedy to cure the long-standing issues and problems associated with L2 learning. The findings reported in this paper suggest that it is the inherent problems of learning per se that prohibit the proliferation of innovative practices of 'digital youth' learners. Specifically, the Chinese undergraduates in the empirical study rarely curated digital resources in an innovative or creation-oriented manner, primarily because behavior as such is not in line with the learning expectations and realities that are rooted in learners' context of learning. As such, the present study has moved beyond the rhetoric of 'digital natives' and hotly debated issue of the 'digital divide' (e.g. the access to and knowledge of digital curation). The present study has revealed the role of context in mediating L2 learners' digital practices in the Chinese university context, and cautioned us against the celebrative and conclusive discourses of digital learning behavior of the 'digital youth'.

\section{References}

Benson, P. and Reinders, H. (Ed.) (2011). Beyond the Language Classroom. Basingstoke: Palgrave Macmillan.

Chen, C. W. (2017): Guided listening with listening journals and curated materials: a metacognitive approach, Innovation in Language Learning and Teaching, DoI: 10.1080/17501229.2017.1381104.

Cresswell, J. W. (2009). Research Design: Qualitative, Quantitative, and Mixed Methods Approach (third edition). Thousand Oaks, CA: Sage.

Crook, C. (2012). The 'digital native' in context: tensions associated with importing Web 2.0 practices into the school setting. Oxford Review of Education, 38(1), 63-80.

Dallas, C. (2015). Digital curation beyond the "wild frontier": a pragmatic approach. Archival Science. DOI: 10.1007/s10502-015-9252-6.

Dooly, M., \& O'Dowd, R. (Eds.). (2012). Researching Online Foreign Language Interaction and Exchange: Theories, Methods and Challenges. Bern: Peter Lang. 
Godwin-Jones, R. (2015). Contributing, Creating, Curating: Digital Literacies for Language Learners. Language Learning \& Technology 19(3), 8-20. Retrieved from http:// llt.msu.edu/issues/october2015/emerging.pdf.

Lai, C. (2014). Perceiving and traversing in-class and out-of-class learning: accounts from foreign language learners in Hong Kong. [Online]. Innovation in Language Learning and Teaching, 1-20. Available at: http://www.tandfonline.com/doi/abs/10 .1080/17501229.2014.918982\#.U-LwVHJO6UQ [Last accessed $11^{\text {th }}$ August 2019].

Jenkins, H., Clinton, K., Purushotma, R., Robison, A. J., \& Weigel, M. (2006). Confronting the challenges of participatory culture: Media education for the 2ist century. Chicago: MacArthur Foundation.

Kaya, T. (2013). Does ICT teacher training bring benefits to the language classroom? In Proceedings of WorldCALL. Glasgow, 10-13 July 2013. [Online]. Available at: http://www.researchgate.net/publication/251232220_Effects_of_online_gaming_ex perience_on_English_achievement_in_an_MMORPG_learning_environment/ file/3deec51ef324086829.pdf\#page $=138$ [Last accessed $11^{\text {th }}$ August 2019].

Kurek, M., \& Hauck, M. (2014). Closing the Digital Divide: A Framework for Multiliteracy Training. In J. Guikema \& L. Williams (Eds.), Digital Literacies in Foreign and Second Language Education (pp. 119-140). San Marcos, TX: Calico.

Lantolf, J. P. (2000). Second language learning as a mediated process. Language Teaching, 33(02), 79-96.

Mihailidis, P. (2015). Digital curation and digital literacy: Evaluating the role of curation in developing critical literacies for participation in digital culture. E-Learning and Digital Media. 12(5-6), 443-458. DoI: 10.1177/2042753016631868.

Potter J., McDougall J. (2017) Digital Curation/Digital Production: Storying the Digital Learner. In Digital Media, Culture and Education. London: Palgrave Macmillan.

Poole, A. H. (2017). "A Greatly Unexplored Area": Digital Curation and Innovation in Digital Humanities. Journal of the Association for Information Science and Technology, 68(7). DOI: 10.1002/asi.23743.

Qian, K., \& Bax, S. (2017). Beyond the language classroom: researching moocs and other innovations (Eds). Dublin: Research-publishing.net.

Raith, T. (2009). 'The use of weblogs in language education'. In M. Thomas (Ed.), Handbook of Research on Web 2.o and Second Language Learning. New York: IG I Global.

Vygotsky, L. S. (2012). Thought and Language. Cambridge, MA: MIт Press.

Zeng, S. (2018). English learning with Web 2.o - An investigation into Chinese undergraduates' technology (non) use and perspectives. Singapore: Springer.

Zhang, J., Lou, X., Zhang, H. \& Zhang, J. (2019). Modeling collective attention in online and flexible learning environments, Distance Education, 40(2), 278-301, DoI: 10.1080/01587919.2019.1600368. 